\title{
EFFECTS OF PSYCHOTROPICS ON NEUTROPHIL-LYMPHOCYTE RATIO IN PSYCHIATRIC PATIENTS
}

\author{
RAJESH VENKATARAMAN*, MUHAMMED RASHID, GUNAKULA AKHILA, SHIJO DANIEL, AMALA THOMAS
}

Department of Pharmacy Practice, Sri Adichunchanagiri College of Pharmacy, Adichunchanagiri University, Mandya, Karnataka, India. Email: rajeshvenky_research@hotmail.com

Received: 27 March 2019, Revised and Accepted: 29 April 2019

\section{ABSTRACT}

Objective: The objective of this study was to investigate whether psychotropic has any effect on neutrophils and lymphocyte ratio (NLR) in psychiatric patients.

Methods: In this hospital-based prospective quasi-randomized follow-up study, we analyzed every 10 patients with newly diagnosed with depression, schizophrenia, and bipolar disorder for complete blood count before and after treatment with the selected psychotropic.

Results: There was a significant increase in NLR of patients with depression after treatment with psychotropic ( $p=0.001$ ), also in schizophrenia, there was a slight increase in NLR of patients after treatment with psychotropic $(\mathrm{p}=0.005)$, but in bipolar disorder, there was a significant decrease in NLR of patients after treatment with psychotropic $(\mathrm{p}=0.002)$.

Conclusion: The present study confirmed that psychotropics have a statistically significant effect on the increase in NLR in patients with depression, a slight increase in patients with schizophrenia, and a decrease in patients with bipolar disorder. Further studies are needed for a better understanding of the mechanism of psychotropic drug effect on NLR.

Keywords: Lymphocytes, Neutrophils, Neutrophils/lymphocyte ratio, Bipolar disorder, Schizophrenia, Depression.

(C) 2019 The Authors. Published by Innovare Academic Sciences Pvt Ltd. This is an open access article under the CC BY license (http://creativecommons. org/licenses/by/4. 0/) DOI: http://dx.doi.org/10.22159/ajpcr.2019.v12i6.33251

\section{INTRODUCTION}

Psychiatric disorders or mental illness is associated with relative changes or distress functioning in social, work, or family activities and characterized by alteration in behavior, emotion, and thinking. The neutrophil-tolymphocyte ratio (NLR) is an inexpensive, reproducible test which can be obtained from the white blood cell count and has been considered as a new biomarker for systemic inflammatory response [1,2]. In some malignancies, increased mortality can be predicted by elevated levels of NLR as it is considered as an important inflammatory marker [3,4]. Depression is a complex disease associated with increased morbidity and is characterized by prominent disabilities, social burden, and reduced quality of life [5]. According to the World Health Organization in 2020, depression will account for the second leading cause for morbidity worldwide [6,7]. Certain studies show that pharmacotherapy for depression has the ability to normalize some of the disturbed indices of immunoreactivity and depression is related to a disturbance in central nervous system (CNS), immune response, and vascular reactivity $[5,8]$.

Schizophrenia is a mental disorder without a satisfactory biological explanation characterized by well-defined symptoms and a lifelong course and by growing body of evidence whose pathophysiology is associated with immunological and inflammatory mechanisms $[9,10]$. The immunopathogenesis mechanism in schizophrenia is evident as several studies discuss the increased permeability of the blood-brain barrier which supports the penetration of T cells to fight an inflammatory CNS process [10].

"Bipolar disorder is a chronic, severe neuropsychiatric disorder with recurrence of mood exacerbation from major depressive episodes to manic episodes and vice versa" and whose lifetime prevalence approximately around 2\% [11]. In 2001, Zahorec developed NLR as an easily measurable and readily available parameter to evaluate the intensity of stress and other systemic inflammations in patients with shock, multiple trauma, major surgery, or sepsis [12]. Bipolar disorder can be explained effectively as a multisystemic inflammatory disease without a proper biological mechanism [11]. The severity of diseases has been examined using scales such as Hamilton depression (HAM-D) for depression, Brief Psychiatric Rating Scale (BPRS) for schizophrenia, and both Young Mania Rating Scale (YMRS) for bipolar disorder. Depressive disorder touches and impairs all aspects of life and it is having a great risk in societal aspects of a human being. It also contributes more to the suicidal thoughts and risk of managing other comorbidities [13].

Most of the psychotropics are associated with dysfunction in sexually related activities, cardiac-related diseases such as arrhythmia, hypotension, and also cardiac death [14]. Leukopenia and agranulocytosis are occasionally noted as side effects of antipsychotic medications. This is particularly true of the antipsychotic medication clozapine, for which the incidence of agranulocytosis is approximately 1\% [12]. According to a study reported by Cramlington, post regarding effects of treatment with carbamazepine monotherapy or in combination with lithium, on leukocyte count significant reductions in total leukocyte, neutrophil, and lymphocyte counts were found with carbamazepine monotherapy, whereas inclusion of lithium with carbamazepine shown marked increases in leukocyte and neutrophil counts but not lymphocyte counts [15].

Psychotropic drugs may also affect the immune system by direct action on immunocompetent cells or indirectly through hormonal secretions. There are no much studies available which assessed the hematological effects of psychotropic, especially the NLR in Indian psychotic patients. We aimed to analyse the effect of psychotropic medication on patient's NLR before, during and after the treatment.

\section{METHODOLOGY}

\section{Study design and setting}

A prospective quasi-randomized follow-up study for 6 months, in which every 10 patients newly diagnosed with depression, schizophrenia, 
and bipolar disorder are admitted, respectively, was conducted after obtaining the ethical approval from IEC, AIMS, B. G Nagara. We considered only antipsychotic drug newer patients. Pediatric patients and patients with infection or other comorbidities were excluded from the study. The severity of disorder was calculated using BPRS, YMRS, and HAM-D scales.

\section{Method}

A total of 30 patients were included in the study. To access the NLR, blood samples were collected from patients and laboratory data were evaluated. The follow-up was done in the duration of 1 month to the patient after administering the medication and their NLR was assessed to evaluate the changes caused by psychotropic. Both the NLRs have been compared to assess the changes in NLR before and after the treatment.

\section{Statistical analysis}

Statistical Package for the Social Sciences (SPSS) acquired by IBM for Windows, Version 17.0. Released Chicago: SPSS Inc; 2009. were used for the development of a data entry sheet and statistical analysis. Descriptive statistics were conducted to demonstrate the demographic characteristics of the subjects. Paired t-test was applied to compare pre- and post-results in both groups. Spearman rank correlation was run to evaluate the correlation between NLR and severity of the disease.

\section{RESULTS}

The mean difference of NLR between baseline and follow-up was statistically significant for depression $(\mathrm{t}=-9.920, \mathrm{p}=0.001$; Fig $1 \mathrm{a}$; Table $1 \mathrm{a})$, schizophrenia ( $\mathrm{t}=-3.682, \mathrm{p}=0.005$; Fig $1 \mathrm{~b}$; Table $1 \mathrm{~b})$, and bipolar disorder $(\mathrm{t}=4.467, \mathrm{p}=0.002$; Fig 1c; Table $1 \mathrm{c})$

In case of patients with depression, when compared to the NLR-baseline, NLR-follow-up was found to be increased, and on Spearman's correlation, NLR-baseline and HAM-D-baseline are negatively correlated with $\mathrm{g}=-0.438$ and $\mathrm{p}=0.206$ (Table $2 \mathrm{a}$ ), whereas NLR-followup and HAM-D-follow-up are positively correlated with $\mathrm{\rho}=0.049$ and $\mathrm{p}=0.894$ (Table $2 \mathrm{~b}$ ) which are not statistically significant. For patients with schizophrenia, NLR-follow-up was higher when compared to NLR- baseline up, and on Spearman's correlation, NLR-baseline and BPRSbaseline are positively correlated with $\mathrm{\rho}=0.231$ and $\mathrm{p}=0.521$ (Table $2 \mathrm{c}$ ), but NLR-follow-up and BPRS-follow-up are negatively correlated with $\mathrm{Q}=-0.219$ and $\mathrm{p}=0.544$ (Table $2 \mathrm{~d}$ ) which are not statistically significant.

In contradiction to the above two groups of patients, NLR-follow-up was lower when compared to NLR-baseline in patients with bipolar disorder. On Spearman's correlation, NLR-baseline and YMRS-follow-up

Table 1a: Comparison of changes in neutrophils and lymphocyte ratio in patients with depression, before and after the treatment

\begin{tabular}{lllll}
\hline Diagnosis & Comparison & Mean \pm SD & t & p \\
\hline Depression & NLR-B & $1.60110 \pm 0.340423$ & -9.920 & $0.001^{* * *}$ \\
& NLR-F & $2.36830 \pm 0.262317$ & & \\
\hline
\end{tabular}

SD: Standard deviation, NLR-B: Neutrophil-lymphocyte ratio baseline,

NLR-F: Neutrophil-lymphocyte ratio follow-up

Table 1b: Comparison of changes in neutrophils and lymphocyte ratio in patients with schizophrenia, before and after the treatment

\begin{tabular}{lllll}
\hline Diagnosis & Comparison & Mean \pm SD & t & p \\
\hline Schizophrenia & NLR-B & $1.86170 \pm 0.342614$ & -3.682 & $0.005^{*}$ \\
& NLR-F & $2.07800 \pm 0.202820$ & & \\
\hline
\end{tabular}

SD: Standard deviation, NLR-B: Neutrophil-lymphocyte ratio baseline,

NLR-F: Neutrophil-lymphocyte ratio follow-up

Table 1c: Comparison of changes in neutrophils and lymphocyte ratio in patients with bipolar disorder, before and after the treatment

\begin{tabular}{lllll}
\hline Diagnosis & Comparison & Mean \pm SD & t & p \\
\hline Bipolar & NLR-B & $3.30830 \pm 0.835098$ & 4.467 & $0.002^{* *}$ \\
disorder & NLR-F & $2.20120 \pm 0.128456$ & & \\
\hline
\end{tabular}

SD: Standard deviation, NLR-B: Neutrophil-lymphocyte ratio baseline, NLR-F: Neutrophil-lymphocyte ratio follow-up

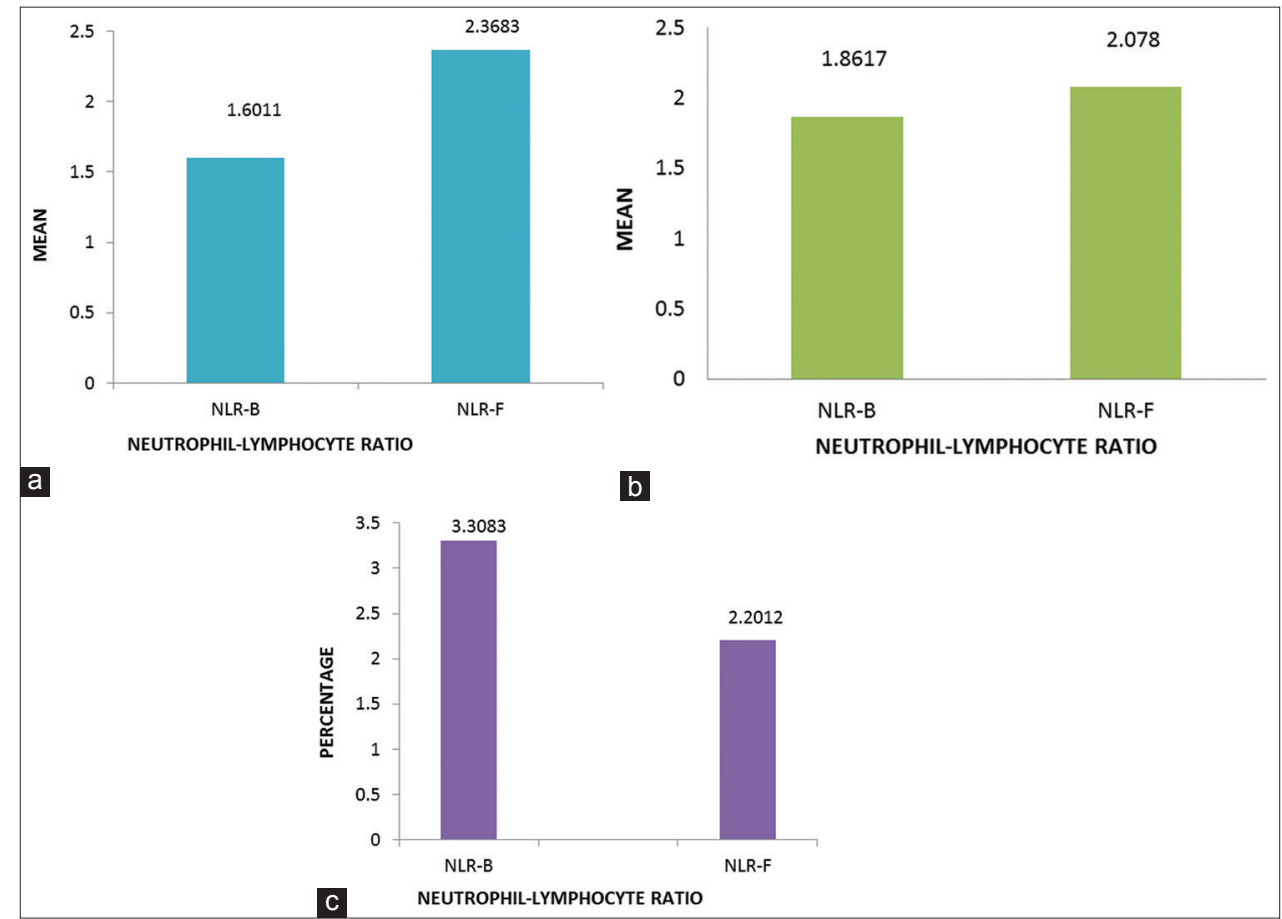

Fig. 1: (a) NLR changes in patients with depression. (b) NLR changes in patients with schizophrenia. (c) NLR changes in patients with bipolar disorder. NLR-B - Neutrophil-lymphocyte ratio baseline. NLR-F - Neutrophil-lymphocyte ratio follow-up 
Table 2a: Correlation between neutrophils and lymphocyte ratio baseline with Hamilton depression scale baseline

\begin{tabular}{llll}
\hline & & & HAM-D baseline \\
\hline Spearman rho & NLR & Correlation & -0.438 \\
& baseline & coefficient & \\
& & $\mathrm{p}$ & 0.206 \\
& & $\mathrm{n}$ & 10 \\
\hline
\end{tabular}

NLR: Neutrophils and lymphocyte ratio, HAM-D: Hamilton depression scale

Table 2b: Correlation between neutrophils and lymphocyte ratio follow-up with Hamilton depression scale follow-up

\begin{tabular}{llll}
\hline & & & HAM-D follow-up \\
\hline Spearman rho & NLR & Correlation & 0.049 \\
& follow-up & coefficient & \\
& & $\mathrm{p}$ & 0.894 \\
& & $\mathrm{n}$ & 10 \\
\hline
\end{tabular}

NLR: Neutrophils and lymphocyte ratio, HAM-D: Hamilton depression scale

Table 2c: Correlation between neutrophils and lymphocyte ratio baseline with brief psychiatric rating scale baseline

\begin{tabular}{llll}
\hline & & & BPRS baseline \\
\hline Spearman rho & $\begin{array}{l}\text { NLR } \\
\text { baseline }\end{array}$ & Correlation & 0.231 \\
& & coefficient & \\
& $\mathrm{p}$ & 0.521 \\
& $\mathrm{n}$ & 10 \\
\hline
\end{tabular}

BPRS: Brief psychiatric rating scale, NLR: Neutrophils and lymphocyte ratio

Table 2d: Correlation between neutrophils and lymphocyte ratio follow-up with brief psychiatric rating scale follow-up

\begin{tabular}{llll}
\hline & & & BPRS follow-up \\
\hline Spearman rho & NLR & Correlation & -0.219 \\
& baseline & coefficient & \\
& & $\mathrm{p}$ & 0.544 \\
& & $\mathrm{n}$ & 10 \\
\hline
\end{tabular}

BPRS: Brief psychiatric rating scale, NLR: Neutrophils and lymphocyte ratio

Table 2e: Correlation between neutrophils and lymphocyte ratio baseline with Young Mania Rating Scale baseline

\begin{tabular}{llll}
\hline & & & YMRS baseline \\
\hline Spearman rho & NLR & Correlation & -0.370 \\
& baseline & coefficient & \\
& & $\mathrm{p}$ & 0.293 \\
& & $\mathrm{n}$ & 10 \\
\hline
\end{tabular}

NLR: Neutrophils and lymphocyte ratio, YMRS: Young Mania Rating Scale

Table 2f: Correlation between neutrophils and lymphocyte ratio follow-up with Young Mania Rating Scale follow-up

\begin{tabular}{llll}
\hline & & & YMRS follow-up \\
\hline Spearman rho & NLR & Correlation & -0.636 \\
& follow-up & coefficient & \\
& & $\mathrm{p}$ & 0.048 \\
& & $\mathrm{n}$ & 10 \\
\hline
\end{tabular}

NLR: Neutrophils and lymphocyte ratio, YMRS: Young Mania Rating Scale

are negatively correlated with $\varrho=-0.370$ and $p=0.293$ (Table 2e) which are not statistically significant and NLR-follow-up and YMRS-follow-up are negatively correlated with $\mathrm{g}=-0.636$ and $\mathrm{p}=0.048$ (Table 2f) which are statistically significant.

\section{DISCUSSION}

The study was aimed to investigate basic data on the effect of psychotropics on NLR. No studies were conducted based on the "effect of psychotropics on NLR in psychiatric patients." This study is the first study in literature conducted based on this topic. Fewer studies are conducted on comparison with changes in NLR and disease condition such as bipolar disorder, schizophrenia, and depression. In this study, majority of subjects belongs to the age group of 19-30 years and in total of $53.4 \%$ of female and $47.6 \%$ of male patients.

In some of the earlier studies, in case of depression, it has been observed that there was no significant difference between the groups based on sociodemographic characteristics such as age, gender, material status, income level, smoking, and employment. Mean NLR value was $1.58 \pm$ 0.59 in the patient group, and it was $2.05 \pm 0.89$ in the control group. The difference between these mean values was statistically significant $(p=0.007)[16]$. However, the mean \pm standard deviation NLR of patients with schizophrenia was significantly higher than that of healthy controls $(2.6 \pm 1.0$ vs. $1.9 \pm 0.7$, respectively, $p<0.001)$ [9]. In bipolar disorder, the NLR was higher in male patients than in female comparison subjects $(3.2 \pm 2.2$ vs. $1.7 \pm 0.4)(p<0.001)$. Furthermore, compared with the healthy male subjects, the male patients had significantly higher NLR $(3.3 \pm 2.4$ vs. $2.0 \pm 0.7)(\mathrm{p}<0.001)$ [17] .

The present study showed that there is a significant increase in the NLR of patients with depression after treatment with psychotropics $(\mathrm{p}=0.001)$. In the case of schizophrenia, there is a slight increase in the NLR of psychiatric patients after treatment with psychotropics $(p=0.005)$. However, in bipolar disorder, there is a significant decrease in the NLR of psychiatric patients after treatment with psychotropics ( $\mathrm{p}=0.002)$. The study has shown that NLR is not significantly correlated with the severity of the diseases in patients with depression, schizophrenia, and bipolar disorder. Although the study includes a lesser number of subjects when compared to related studies, it might obtain certain preliminary data regarding the effects of psychotropic in NLR. However, the study has not carried out for more number of psychotropic.

\section{CONCLUSION}

The study is the first to examine the effect of psychotropic on such an inexpensive biomarker like NLR. It was found that decreased NLR was get increased in patients with depression and schizophrenia after treatment with psychotropic and in case of bipolar disorder elevated level get reduced. According to findings, psychotropic has a certain effect on NLR in the drug newer psychiatric patients. Further studies can be carried out in patients administered with individual psychotropic.

\section{ACKNOWLEDGMENT}

Patients and their caretakers are highly acknowledged by the authors for their kind support and cooperation.

\section{AUTHORS' CONTRIBUTIONS}

All authors contributed in one or the other stages of designing the study, conducting the study, statistical analysis, interpreting the results, and/or writing the manuscript. Furthermore, all authors reviewed the final manuscript and accepted for the publication.

\section{FUNDING}

No funding is received from any organization or society.

\section{CONFLICTS OF INTEREST}

Authors declare that there are no conflicts of interest.

\section{REFERENCES}

1. Sunbul M, Gerin F, Durmus E, Kivrak T, Sari I, Tigen K, et al. Neutrophil to lymphocyte and platelet to lymphocyte ratio in patients with dipper 
versus non-dipper hypertension. Clin Exp Hypertens 2014;36:217-21.

2. Gibson PH, Cuthbertson BH, Croal BL, Rae D, El-Shafei H, Gibson G, et al. Usefulness of neutrophil/lymphocyte ratio as predictor of newonset atrial fibrillation after coronary artery bypass grafting. Am J Cardiol 2010;105:186-91.

3. Halazun KJ, Aldoori A, Malik HZ, Al-Mukhtar A, Prasad KR, Toogood GJ, et al. Elevated preoperative neutrophil to lymphocyte ratio predicts survival following hepatic resection for colorectal liver metastases. Eur J Surg Oncol 2008;34:55-60.

4. Tamhane UU, Aneja S, Montgomery D, Rogers EK, Eagle KA, Gurm HS, et al. Association between admission neutrophil to lymphocyte ratio and outcomes in patients with acute coronary syndrome. Am J Cardiol 2008;102:653-7.

5. Aydin Sunbul E, Sunbul M, Yanartas O, Cengiz F, Bozbay M, Sari I, et al. Increased neutrophil/Lymphocyte ratio in patients with depression is correlated with the severity of depression and cardiovascular risk factors. Psychiatry Investig 2016;13:121-6.

6. Ebmeier KP, Donaghey C, Steele JD, Association AP, Kessler R, Berglund $\mathrm{P}$, et al. Recent developments and current controversies in depression. Lancet (London, England) 2006;367:153-67.

7. World Health Organization. The World Health Report: 2001. Mental Health: New Understanding, New Hope. Geneva: World Health Organization; 2001.

8. Canan F, Ataoglu A. Effect of escitalopram on white blood cells in patients with major depression. J Clin Med Res 2009;1:290-1.

9. Semiz M, Yildirim O, Canan F, Demir S, Hasbek E, Tuman TC, et al. Elevated neutrophil/lymphocyte ratio in patients with schizophrenia.
Psychiatr Danub 2014;26:220-5.

10. Rothermundt M, Arolt V, Bayer TA. Review of immunological and immunopathological findings in schizophrenia. Brain Behav Immun 2001;15:319-39.

11. Ayhan MG, Cicek IE, Inanli I, Caliskan AM, Ercan SK, Eren I. Neutrophil/ lymphocyte and platelet/lymphocyte ratios in all mood states of bipolar disorder. Psychiatry Clin Psychopharmacol 2017;27:284-8. Available from:http://www.ovidsp.ovid.com/ovidweb.cgi?T=JS\& PAGE=reference $\& \mathrm{D}=\mathrm{psyc} 14 \& \mathrm{NEWS}=\mathrm{N} \& \mathrm{AN}=2018-16725-012$

12. Cakır U, Tuman TC, Yıldırım O. Increased neutrophil/lymphoctye ratio in patients with bipolar disorder: A preliminary study. Psychiatr Danub 2015;27:180-4.

13. Zam WI. Vitamin D deficiency and depressive disorders: Review study of probable relationship. Int J Pharm Pharm Sci 2016;8:16-22.

14. Ilyaz M, Baig MM, Quadir MA, Fathima M, Khan SA. Drug utilization study of antipsychotics and its common ADR'S in the psychiatry OPD of OHRC. Int J Pharm Pharm Sci 2014;6:162-5.

15. Oyewumi LK, McKnight M, Cernovsky ZZ. Lithium dosage and leukocyte counts in psychiatric patients. J Psychiatry Neurosci 1999;24:215-21.

16. Korkmaz S, Denk A, Gundogani B, Korucu T, Dulkadir ZE, Telo MS. Atmaca. neutrophil lymphocyte ratio in patients with major depressive disorder. Acta Med Mediterr 2016;32:795-8.

17. Çakır U, Tuman TC, Yıldırım O. Increased neutrophil/lymphoctye ratio in patients with bipolar disorder: A preliminary study. Psychiatr Danub 2015;27:180-4. Available from: http://www.ncbi.nlm.nih.gov/ pubmed/26057314 